\title{
Destruction of localized electron pairs above the magnetic-field-driven superconductor-insulator transition in amorphous InO films
}

\author{
V.F. Gantmakher*, M.V. Golubkov, V.T. Dolgopolov, G.E. Tsydynzhapov, and A.A. Shashkin \\ Institute of Solid State Physics, Russian Academy of Sciences, 142432 Chernogolovka, Russia
}

\begin{abstract}
We have investigated the field-induced superconductivity-destroying quantum transition in amorphous indium oxide films at low temperatures down to $30 \mathrm{mK}$. It has been found that, on the high-field side of the transition, the magnetoresistance reaches a maximum and the phase can be insulating as well as metallic. With further increasing magnetic field the film resistance drops and approaches in the high-field limit the resistance value at transition point so that at high fields the metallic phase occurs for both cases. We give a qualitative account of this behavior in terms of field-induced destruction of localized electron pairs.
\end{abstract}

The theoretical description of the zero-field and fieldinduced quantum superconductor-insulator transitions (SIT) in a $2 \mathrm{D}$ superconductor is based on a concept of electron pairs which are delocalized on the superconducting side and localized on the insulating side of transition [1] 3]. According to Refs. [1] 3], the temperature dependence of the film resistance near the field-induced SIT is controlled by deviation $\delta=B-B_{c}$ from the critical field $B_{c}$ and the most specific among perceptible features of SIT is fan-like set of resistance-vs-temperature curves $R_{\delta}(T)$. Such a set is expected to collapse onto a single curve as a function of scaling variable $\delta / T^{1 / y}$, where $y$ is the critical index, see review 任. Many of the SIT studies were performed on amorphous $\operatorname{In}_{2} \mathrm{O}_{x}(x<3)$ films whose conductivity is caused by oxygen deficiency compared to fully stoichiometric insulating compound $\operatorname{In}_{2} \mathrm{O}_{3}$ : by changing the oxygen content one can cover the range from a superconductor to an insulator and thus realize the zero-field SIT. On the insulating side of this SIT, observation was reported of the activation behavior of the resistance $R \propto \exp \left(T_{0} / T\right)^{p}$ with $p=1$ (Arrhenius law) and activation energy $T_{0}$ tending to zero as the phase boundary is approached [5]. It was found later that switching a magnetic field results in decreasing the resistance and weakening its temperature dependence from the Arrhenius law to the Mott law with exponent $p=1 / 4$ [6]. This was explained in Ref. [6] by magnetic-fieldcaused suppression of the binding energy $\Delta$ of localized electron pairs which manifests as a gap at the Fermi level.

Field-induced SIT is realized on the superconducting side of zero-field SIT. It is indicated by fan-like structure of experimental curves $R_{\delta}(T)$ such that, in accordance with the scaling analysis, the expected collapse is indeed the case [7]. Above the field-induced SIT, the existence of two insulating phases was postulated based on results of Hall measurements [8]; however, temperature dependences of the resistance of these phases were not studied. Reversal of a zero-bias peak in the differential resistance at the critical field $B_{c}$ was observed and attributed to the granular structure of films [9].
TABLE I. Parameters of two states of the sample. $R_{r}$ is the resistance at room temperature, the values of $R_{c}$ and $B_{c}$ are determined by means of scaling analysis as described in Ref. [11].

\begin{tabular}{l|ccc}
\hline \hline State & $R_{r}, \mathrm{k} \Omega$ & $R_{c}, \mathrm{k} \Omega$ & $B_{c}, \mathrm{~T}$ \\
\hline 1 & 3.4 & 7.5 & 2 \\
2 & 3.0 & 9.2 & 7.2 \\
\hline \hline
\end{tabular}

Here, we investigate the phase on the high-field side of SIT where the occurrence of localized electron pairs is predicted. We find that, while this phase can be both insulating and metallic, in the high-field limit the system always enters the metallic phase. This is interpreted as field-caused breaking of localized electron pairs.

The experiments were performed on a $200 \AA$ thick amorphous $\operatorname{In}_{2} \mathrm{O}_{x}(x<3)$ film without pronounced granularity as was checked by the absence of quasireentrant transition, i.e., the absence of minimum on dependences $R_{\delta}(T)$ at low temperatures [10]. The oxygen content $x$ could be reversibly altered by heat treatment; all experimental procedures are described in detail in Ref. [6]. Assuming for the sake of simplicity that the film disorder remains unchanged during heat treatment, the quantity $x$ controls the carrier density $n$ and, then, it is the variation of $n$ that causes zero-field SIT. Two states of the film were studied with parameters listed in Table 1 . Under the above assumption, the carrier density in a state should be inversely proportional to its room temperature resistance. Hence, state 2 is farther from the zero-field SIT and deeper in the superconducting phase as compared to state 1 . The magnetoresistance of both states was measured in Oxford TLM-400 dilution refrigerator in the temperature range $1.2 \mathrm{~K}$ to $30 \mathrm{mK}$ using a four-terminal lock-in technique at a frequency of $10 \mathrm{~Hz}$. The current across the sample was equal to $5 \mathrm{nA}$ and corresponded to the linear regime of response. The measurement runs were made by sweeping magnetic field at fixed temperature.

Our preceding study has confirmed the existence of 


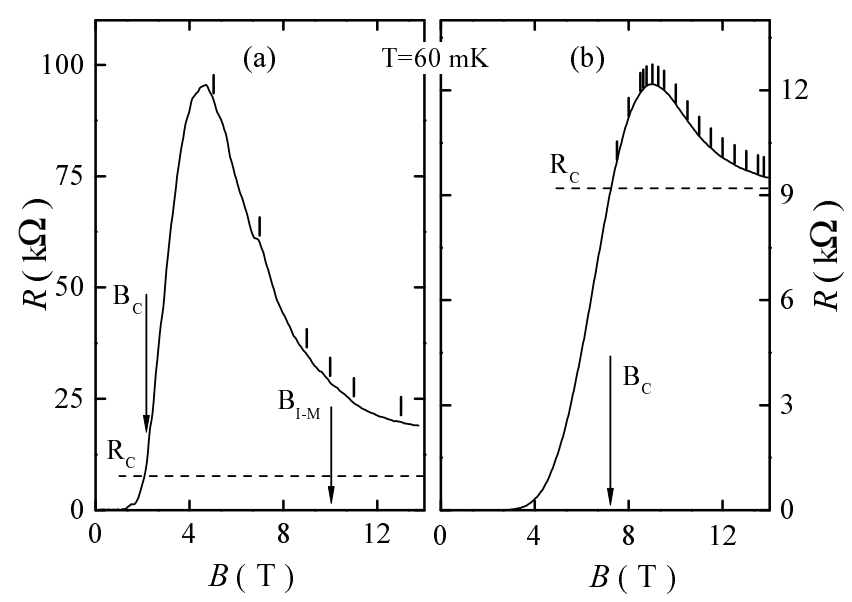

FIG. 1. Magnetoresistance of the film in state 1 (a) and in state $2(\mathrm{~b})$. The critical $R_{c}$ and $B_{c}$ values at $T=0$ are indicated. Also shown is the position of metal-insulator transition, $B_{I-M}$, determined from Fig. 2. The temperature dependences of the resistance are analyzed at fields marked by vertical bars.

magnetic-field-tuned quantum SIT in such films and revealed that this phenomenon is more general compared to the one considered in Ref. [2]. Particularly, to attain collapse of data $R_{\delta}(T)$ in the vicinity of transition against scaling variable $\delta / T^{1 / y}$ account should be taken, e.g., of the temperature dependence of the critical resistance $R_{c}$, which gives rise to the appearance of a linear term in $T$ in dependences $R_{\delta}(T)$ [11].

Fig. 1 displays the magnetoresistance traces for both states of the film at a temperature of $60 \mathrm{mK}$. The critical field $B_{c}$ and resistance $R_{c}$ at $T=0$ (Table II) are determined with the help of scaling analysis as described in detail in Ref. [11. One can see from the figure that with increasing field the magnetoresistance for both of the states reaches a maximum $R_{\max }$ above $B_{c}$ and then drops so that in the high-field limit it approaches the value of $R_{c}$. The relative value of maximum $R_{\max } / R_{c}$ is considerably larger for state 1 which is closer to the zero-field SIT; moreover, the phase right above $B_{c}$ is insulating in state 1 and metallic in state 2 , as will be shown below.

The vertical bars in Fig. 1 1 mark the magnetic field values at which the temperature dependence of resistance is analyzed. The results of such an analysis for state 1 are represented in Fig. 2. At fields near the resistance maximum $R(T)$ follows the activation behavior as expected for an insulator (inset to Fig. 2). However, at higher fields the activation law does not hold, nor do the logarithmic corrections normally observed in 2D metals [12]. That is why we examine the film resistance over the field range 7 to $13 \mathrm{~T}$ in terms of $3 \mathrm{D}$ material behavior in the

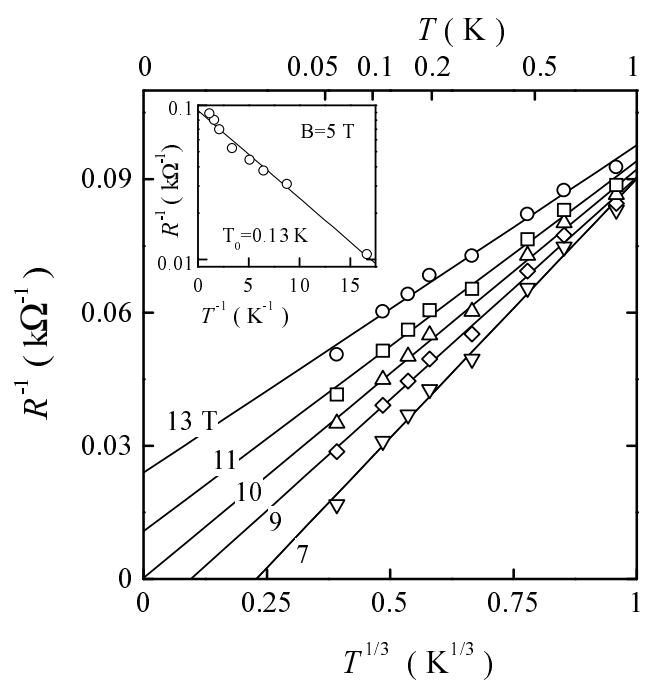

FIG. 2. Temperature dependence of the high-field conductance of state 1 at various magnetic fields. Arrhenius plot of the conductance at $B=5 \mathrm{~T}$ is displayed in the inset.

vicinity of metal-insulator transition [13, 14]:

$$
\sigma(T)=a+b T^{1 / 3} \quad b>0,
$$

where the sign of the parameter $a$ discriminates between a metal and an insulator at $T \rightarrow 0$. If $a>0$, it yields zero temperature conductivity $\sigma(0)=a$, whereas the negative $a$ points to activated conductance at lower temperatures.

We emphasize that we judge about the transport properties at $T=0$ as obtained by extrapolation from above $30 \mathrm{mK}$. Bearing this in mind, we determine from Fig. 2 the field $B_{I-M} \approx 10 \mathrm{~T}$ of metal-insulator transition for state 1. So, a conclusion of Ref. [8] about the presence of two phases above the SIT is confirmed. Yet, in contrast to Ref. [8], we find that their phase boundary is not near the resistance maximum but at appreciably higher field and that the high-field phase is metallic (Fig. 1).

For state 2 the parameter $a$ is positive over the entire field range 7.5 to $14 \mathrm{~T}$ above $B_{c}$ so that there is no insulating phase. The corresponding field dependence of $R_{0} \equiv R_{\delta}(0)=1 / a$ is presented in Fig. 3 alongside with the experimental curves $R(B)$ at 30 and $300 \mathrm{mK}$. Although the extrapolation is far, the tendency for the lowest temperature data to approach $R_{c}$ in the high-field limit seems valid for the extrapolated dependence as well.

The rise of the resistance near the field-driven quantum SIT is in agreement with theoretical ideas about localized electron pairs: above $B_{c}$ it reflects the decrease of the pair localization length $\xi_{\text {loc }}$ with increasing $B$ [1 -3 . That the resistance reaches a maximum with further increasing $B$ was not discussed theoretically so far. Nevertheless, a qualitative account of the observed resistance drop with field can be given in terms of pair breaking caused by 


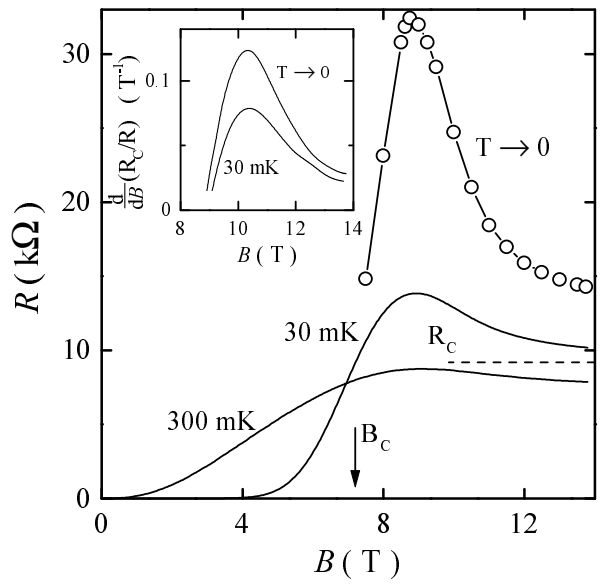

FIG. 3. Magnetoresistance of the film in state 2 at $T=30$ and $300 \mathrm{mK}$, and for $T \rightarrow 0$ as obtained from extrapolations (circles) in accordance with Eq. (1). The critical field and resistance are indicated. The field derivatives of $R_{c} / R$ for $T \rightarrow 0$ and $T=30 \mathrm{mK}$ are compared in the inset.

magnetic field [6]. In this case the behavior of the system of depaired electrons is naturally determined by their density $n_{d}(B)$ : at low $n_{d}$ depaired electrons are certainly localized whereas at sufficiently high $n_{d}$ a metal-insulator transition may be expected. It is the second conduction channel that allows interpretation of the observed nonmonotonic dependences of magnetoresistance.

In agreement with experiment, Fig. 1/ schematically shows the field behavior for three states of the sample. Two of these, $\alpha$ and $\beta$, that are selected in the superconducting phase above the zero-field SIT at $n>n_{c}$ correspond to the studied states 1 and 2, respectively. State $\gamma$ selected in the insulating phase at $n<n_{c}$ corresponds to samples from Ref. [6]. With increasing $B$ state $\alpha$ undergoes the field-induced SIT at $B=B_{c}$ so that the depaired electrons available are localized at that small density $n_{d}$ (Fig. (1). With further increase of field the value of $n_{d}$ enhances due to localized pair breaking, giving rise to the metal-insulator transition in the system of depaired electrons. At $B \rightarrow \infty$, all electron pairs are expected to be broken and so the value of $n_{d}$ should be equal to the carrier density $n$. The different behavior of state $\beta$ is due to larger density $n_{d}$ at the field-induced SIT because of higher $n$ and $B_{c}$. As a result, the depaired electrons are already delocalized at $B=B_{c}$ and thus the field range of the insulating phase shrinks as the

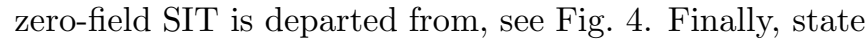
$\gamma$ approaches the metal-insulator phase boundary with increasing $B$ but remains insulating for all fields [6].

Thus, the concept of field-induced pair breaking requires the additional assumption that a metal-insulator transition occurs in the system of depaired electrons.
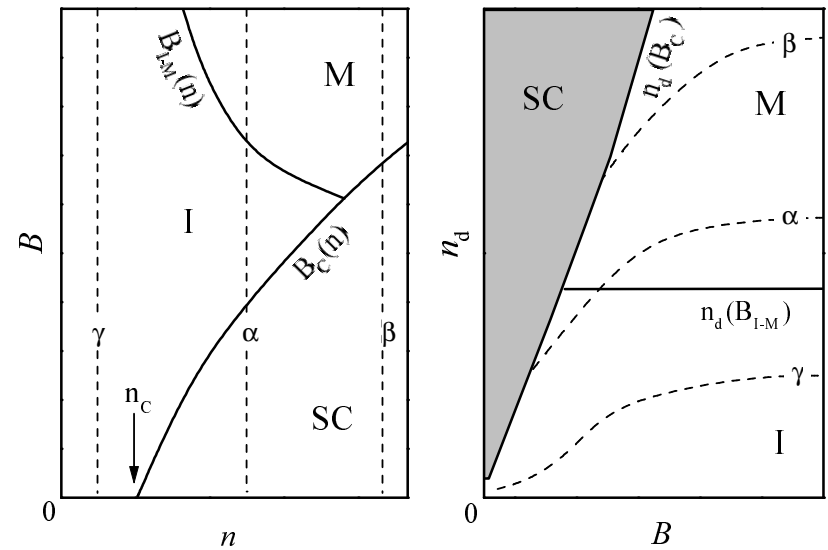

FIG. 4. Schematic phase diagram of the observed transitions in the $(n, B)$ and $\left(B, n_{d}\right)$ planes. The evolution of states $\alpha, \beta, \gamma$ with magnetic field is shown by dashed lines. In shaded area the value $n_{d}$ is not defined.

Also, a theory [1 [3] should be extended to include the possibility of a direct superconductor-metal quantum transition.

Although the origin of localized electron pairs is still an open question, a likely candidate for their breaking might be the paramagnetic effect. In this case the pair breaking field should be proportional to the binding energy of a pair $B^{*}=2 \Delta / g \mu_{B}$, where $g$ is the Landé factor. It is clear that the broad field interval of the negative differential magnetoresistance points to wide dispersion of the pair binding energies. To estimate the distribution function $\nu(\Delta)$ for state 2 we presume for the sake of simplicity that, at fields above the resistance maximum, $R_{0}(B)$ at $T \rightarrow 0$ is inversely proportional to the density of depaired electrons (the Drude limit)

$$
R_{c} / R_{0}=n_{d} / n
$$

where $n$ is the carrier density in the metallic state at $B \rightarrow \infty$, and $n_{d}$ is given by the formula

$$
n_{d}=n-2 \int_{g \mu_{B} B / 2}^{\infty} \nu(\Delta) \mathrm{d} \Delta .
$$

Then, it is easy to obtain the distribution function

$$
\nu(\Delta)=\left.\frac{2 n}{g \mu_{B}} \frac{\mathrm{d}}{\mathrm{d} B}\left(R_{c} / R_{0}\right)\right|_{B=2 \Delta / g \mu_{B}} .
$$

The field derivative of the ratio $R_{c} / R_{0}$, which is proportional to $\nu(B)$, is depicted in the inset of Fig. 3 . Its behavior is similar to that of the field derivative of $R_{c} / R(T=30 \mathrm{mK})$ in spite of the far extrapolation to get $R_{0}(B)$, see Fig. 3 . 
The fact that the distribution $\nu(\Delta)$ is broad allows us to distinguish between two localization scenarios of electron pairs: (i) the localization radius $\xi_{l o c}$ is larger than the pair size $\xi_{0}$; and (ii) $\xi_{l o c}<\xi_{0}$. In the first case the binding energy $\Delta$ is determined mainly by intrinsic factors and is expected to be approximately the same for all pairs. In the opposite case two electrons forming a pair are localized at separate sites and so the binding energy of the pair depends crucially on local random potential [6]. This implies that the dispersion of $\Delta$ is broad. Hence, the obtained data are likely to point to the second scenario of localization. We note that the limit $\xi_{l o c}<\xi_{0}$ was assumed in a model of localized bipolarons [15].

In summary, our study of the field-driven quantum SIT in amorphous $\mathrm{In}_{2} \mathrm{O}_{x}$ films shows that, on the high-field side of the transition, with increasing $B$ the film magnetoresistance reaches a maximum and then drops approaching in the high-field limit the resistance $R_{c}$ at transition point. We find that the high-field phase is always metallic while the phase right above $B_{c}$ can be both insulating and metallic, dependent on distance to the zerofield SIT. The obtained experimental data can be understood within a model of localized electron pairs if one includes (i) a concept of field-caused pair breaking that presumes a metal-insulator transition in the system of depaired electrons; and (ii) a meaning of superconductormetal quantum transition. That the negative differential magnetoresistance is observed in a wide field region is likely to point to broad dispersion of the binding energies of localized electron pairs.

This work was supported by Grants RFBR 96-0217497, RFBR 97-02-16829, INTAS-RFBR 95-302 and by the Programme "Statistical Physics" from the Russian Ministry of Sciences.

[1] M.P.A. Fisher, G. Grinshtein, and S.M. Girvin, Phys. Rev. Lett. 64, 587 (1990).

[2] M.P.A. Fisher, Phys. Rev. Lett. 65, 923 (1990).

[3] S.M. Girvin, M. Wallin, M.-C. Cha, et al., Prog. Theor. Phys. Suppl. 107, 135 (1992).

[4] S.L. Sondhi, S.M. Girvin, J.P. Carini, and D. Shahar, Rev. Mod. Phys. 69, 315 (1997).

[5] D. Shahar, and Z. Ovadyahu, Phys. Rev. B 46, 10917 (1992).

[6] V.F. Gantmakher, M.V. Golubkov, J.G.S. Lok, and A.K. Geim, JETP 82, 951 (1996).

[7] A.F. Hebard, and M.A. Paalanen, Phys. Rev. Lett. 65, 927 (1990).

[8] M.A. Paalanen, A.F. Hebard, and R.R. Ruel, Phys. Rev. Lett. 69, 1604 (1992).

[9] K. Kim, and H.-L. Lee, Phys. Rev. B 54, 13152 (1996).

[10] Y. Liu, D.B. Haviland, B. Nease, and A.M. Goldman,
Phys. Rev. B 47, 5931 (1993).

[11] V.F. Gantmakher, M.V. Golubkov, V.T. Dolgopolov, G.E. Tsydynzhapov, and A.A. Shashkin, condmat/9806244.

[12] G. Bergmann, Phys. Rep. 107, 1 (1984).

[13] Y. Imry, and Z. Ovadyahu, J. Phys. C 15, L327 (1982).

[14] V.F. Gantmakher, V.N. Zverev, V.M. Teplinskii, and O.I. Barkalov, JETP 76, 714 (1993).

[15] A.S. Alexandrov, and N.F. Mott, Rep. Prog. Phys. 57, 1197 (1994). 